\title{
Update on Spinal Cord Stimulation for Chronic Pain
}

\author{
Moo Seong Kim1, Jung Yul Park \\ ${ }^{1}$ Department of Neurosurgery, Busan Paik Hospital, Inje University College of Medicine, Busan, Korea \\ ${ }^{2}$ Department of Neurosurgery, Anam Hospital, Korea University College of Medicine, Seoul, Korea
}

\begin{abstract}
Theoretical basis of the spinal cord stimulation (SCS) is well-known gate control theory for segmental pain suppression. Its general concept is to apply electrical stimulation to the large diameter afferents (producing paresthesia) in the dorsal columns of the spinal cord, which via the central collaterals connect to the "gating mechanisms" in the dorsal horns (DH), thereby inhibiting the pain transmission by smaller diameter afferents. Spinal cord stimulation is generally indicated for the chronic, disabling pain that is refractory to other types of treatments. Common clinical indications for SCS are those clinical pain syndromes associated with neuropathic pain such as failed back surgery syndrome, complex regional pain syndromes, cancer pain, and pain associated with spasticity. Other types of pain associated with tissue ischemia attributable to peripheral vascular disease and intractable angina pectoris appears to be the rewarding indication for spinal cord stimulation. Nowadays, there has been introduction of new hardwares and stimulation techniques based on extensive basic and clinical research such as rechargeable generators, multicolumn electrode leads, independent current delivery, percutaneously insertable paddle leads, long-range telemetry, self-adjustable stimulation, magnetic resonance imaging compatibility, and high with burst frequency stimulation. The purpose of this review is to introduce the new paradigm of spinal cord stimulation with up-to-dated knowledge on basic and clinical research with emphasis on new stimulation indications, techniques with new device, and new targets for the stimulation.
\end{abstract}

Key Words: Spinal cord stimulation; Indication; Burst stimulation; Target; Device

$\triangle$ Corresponding Author: Jung Yul Park, Department of Neurosurgery, Anam Hospital, Korea University College of Medicine, 73 Inchon-ro, Sungbuk-gu, Seoul 02841, Korea. Tel: +82-2-920-5729, Fax: +82-2-929-0629, E-mail: jypark98@korea.ac.kr

\section{INTRODUCTION}

The presupposed basis for the pain-relieving effect of SCS according to the gate control theory was that nociceptive input from the periphery could be inhibited at the first dorsal horn relay by stimulation-induced antidromic activation of collaterals of large dorsal column fibers projecting out to the same spinal segment. A supplementary study demonstrated that the reduction of mechanical hypersensitivity could be produced by SCS recruiting only a small fraction of antidromically activated dorsal column $\mathrm{A} \beta$ fibers $^{43)}$. When applied for neuropathic pain, intermittent SCS for 30 minutes' duration may produce several hours of pain relief after the stimulus is off, indicating long-lasting modulation of neuronal activity ${ }^{43}$.

This long-term effect presumably reflects changes in the local transmitter systems from dorsal horn or in supraspinal loci. Data from humans on biochemical studies correlate to the beneficial effects of SCS are sparse and partly contradictory. Some animal experimental studies showed that SCS was accompanied by a release of gamma-aminobutyric acid (GABA), acetylcholine, adenosine and serotonin in the DH. It should be noted that there are no intraspinal serotonergic neuronal cell bodies, and all the spinal serotonin originates from terminals of cell bodies, in the nucleus raphe magnus complex projecting caudally to the dorsal horn.

The involvement of supraspinal mechanism that Rees and Roberts focused on the possible role of the anterior pretectal nucleus in the SCS effects ${ }^{25}$. They demonstrated that stimulation of the dorsal coulmns could excite cells in the anterior pretectal nucleus, where a profound analgesia by the inhibition of nociceptive dorsal horn neurons may be produced. In a recent fMRI study the most prominent changes related to SCS induced pain relief appeared as a deactivation in the medial thalamus and the rostral anterior cingulate cortex ${ }^{30)}$.

Newly introduced stimulation algorithms are high frequency kilohertz (up to $10 \mathrm{KHz}$ ) of short-duration, biphasic pulses. So far, the reported clinical outcomes are inconsistent. However, there are no data indicating that SCS applied with high frequencies is associated with disturbance of tactile sensibility, and therefore it is difficult to conceptualize the underlying physiologic mechanisms ${ }^{8,41}$. Second is the use of a bursting stimulation (internal frequency $500 \mathrm{~Hz}$; burst delivered with $40 \mathrm{~Hz}$ ) of SCS also aiming at subparesthetic stimulation ${ }^{10)}$. Although new technique of stimulation has shown to be effective and useful in that it would not cause "induced paresthesia" and thus help patients to experience untoward unpleasant feeling during stimu- 
lation, it still needs long-term follow up evaluation since it only has been available for less than two years in clinical setting. From current standpoint of expectation its future seems bright and promising.

\section{RESULTS}

Based on up-to-dated search on spinal cord stimulation, the current status of stimulating electrodes and types of stimulation are described as followings.

\section{Electrodes}

There are $4,8,16,20$, and 32 contacts, electrodes are 1 column, 2, 3, 4 and 5 columns. Electrodes can be classified according to their shape (cylindrical or plate/paddle) or implantation technique (percutaneous or laminectomy). One 16contact percutaneous electrode, the Infinion (Boston Scientific, Valencia, CA, USA), is available. The simple paddle electrode is quadripolar (e.g. the Medtronic [Minneapolis, MN, USA], Resume or Resume-TL, the St. Jude Lamitrode), with four contacts arranged linearly on a single paddle ${ }^{19}$.

Electrodes with 8 or 16 contacts increase the potential for stimulating multiple targets. These electrodes include the specify 3,998 and the specify $2 \times 8$ (both Medtonic), the Lamitrode 44 and 88 (both St. Jude Neuromodulation), and the Artisan (Boston Scientific) ${ }^{19}$. Coupled with a "dual channel" control system, these electrodes allow great flexibility of stimulation. Some paddle electrodes have more than two columns of contacts, such as the St. Jude Penta (five columns) and the Medtronic specify 3,999 (three columns) ${ }^{19)}$.

\section{Types of Stimulations}

\section{1) Extraspinal stimulation}

There are peripheral nerve stimulation, peripheral field stimulation, dorsal ganglion stimulation and nerve root stimulation.

The subcutaneous stimulation of end branches of peripheral nerves is often referred to as "field" stimulation or PNfS to distinguish this form of stimulation from that of peripheral "named" nerves. Whereas stimulation of a specific nerve induces paresthesia along the innervated territory, field stimulation produces paresthesias along a diffuse painful area that may not correlate with one specific dermatome or otherwise be welldefined. For the use of PNfS in the head and scalp, leads are placed subcutaneously in an area of scalp surrounding the painful region to generate a large region of perceived paresthesia that results from stimulation of small cutaneous peripheral nerve fibers within the electrical field.

Another type is the stimulation of dorsal root ganglion (DRG) - another intraspinal structure that may be reached via a trans- spinal of the peripheral nervous system, its anatomical proximity to the spinal canal is likely to keep DRG stimulation grouped together with SCS in a "spinal stimulation" approach. A cluster of bodies of the primary sensory neurons, the DRG is encased by the dura and located inside each intervertebral foramen. In the past, the DRG was the target of destructive surgical intervention, so-called ganglionectomy, which is presently abandoned, partially because of the success of SCS, which became the procedure of choice for patients with failed back surgery syndrome and persistent radiculopathy. However, there are many advantages in targeting DRG with electrical stimulation, not only because it has been implicated in the development and maintenance of chronic pain, but also because it is relatively immobile owing to its anatomic location and is surrounded by a much thinner (compared with the spinal cord) layer of cerebrospinal fluid.

DRG stimulation was the subject of a recent multicenter, prospective study ${ }^{17}$; of 51 patients screened, 39 obtained $>50 \%$ improvement in pain levels during the trial, 32 of whom proceeded with permanent implantation and were followed up for 6 months. Of these, more than 50\% reduction in pain in the back, leg, and foot 6 months after implantation were observed in $57 \%, 70 \%$, and $89 \%$ of patients, respectively. The ability to capture discrete painful areas (such as feet) and stable paresthesia intensities across the body positions were additional benefits encountered in the study ${ }^{17}$.

A prospective, randomized, controlled study of DRG stimulation is currently underway in multiple centers in the USA $(\mathrm{NCT} 1923985)^{177}$. It evaluates the safety and effectiveness of the dedicated DRG stimulation system for the treatment of chronic lower limb pain in persons diagnosed with complex regional pain syndrome or peripheral causalgia compared with conventional SCS in terms of more than 50\% pain relief and incidence of stimulation-related neurological deficits ${ }^{17}$.

\section{2) Hybrid neurostimulation}

A newly introduced type of stimulation method is combination of spinal cord stimulation and peripheral nerve stimulation, called the "hybrid" neurostimulation approach ${ }^{25}$. The majority of patients in both parts of the study chose combined stimulation, with a overall success rate of $85-90 \%$ in terms of axial pain relief ${ }^{29)}$. Another interesting concept was recently presented through a retrospective study that evaluated the benefits of combined spinal cord and PNFS in a group of 40 patients followed up for 6 months after the implantation ${ }^{31}$. The study showed that so called "triangular" stimulation, with the SCS lead set as an anode and the PNFS leads set as cathodes, was a performed program type in $>50 \%$ of the patients ${ }^{31)}$.

\section{3) Burst stimulation}

This type of stimulation delivers a short train of closely 
spaced high frequency stimuli to the spinal cord. Each train of impulses includes five 1 ms-wide spikes with a 1-ms spike interval at a rate of $500 / \mathrm{s}(500 \mathrm{~Hz}$ spike mode). These trains are delivered 40 times per second resulting in a $40-\mathrm{Hz}$ burst mode ${ }^{11)}$. It can be hypothesized that burst stimulation not only modulates the lateral discriminatory pain system but also the medial affective/attentional pain system.

Pain stimuli are indeed processed in parallel ${ }^{15)}$ by two pathways: a medial affective/attentional pain pathway and a lateral discriminatory pathway ${ }^{22,33,34)}$. The medial system is triggered by nociceptive-specific neurons, firing in burst mode, and relayed in lamina I of the spinal horn to the mediodorsal and ventromedial nucleus of the thalamus and from there to the anterior cingulated cortex, anterior insula, and amygdala. The lateral system is triggered predominantly by the wide dynamic range neurons, firing in tonic mode and relaying in lamina I and IV-VI of the dorsal horn to the VPL and VPM nucleus of the thalamus and from there to the primary and secondary somatosensory cortex, posterior parietal area ${ }^{5,26,33)}$.

The reason why burst stimulation does not generate more paresthesias than placebo is unknown, but a hypothesis has been forwarded ${ }^{11)}$. One potential explanation is that the charge per pulse does not differ significantly between burst and tonic stimulation, even though the amplitude is significantly lower. This is most likely because of the larger pulse width of the burst design and the lower amplitudes delivered with burst stimulation ${ }^{11)}$ could induce subthreshold stimulation of the the $A \beta$ fibers, which have been implicated in the generation of paresthesia $^{32)}$. Burst stimulation could therefore already suppress pain via the electrophysiological gate control mechanism before the clinical paresthesia threshold is reached. This hypothesis should be verified by further neuroscientific research.

The results of this study were obtained after a short-term evaluation. However, the first, albeit-uncontrolled, study has shown that the results remain very stable in patients for at least 2 years, permitting us to draw firm conclusions that are very likely stable and robust. Ridder et al. ${ }^{36}$ demonstrated that burst SCS was capable of suppressing neuropathic pain better than placebo in a statistically significant and clinically relevant way, possibly because burst stimulation modulates the medial pain system.

\section{4) Neuromodulation advanced stimulation}

Stimulation is delivered as a charge-balanced pulses from an IPG powered by a battery through lead(s) to electrode contacts in the epidural space. Some SCS systems contain a non-rechargeable lithium battery: others contain a transcutaneously rechargeable battery.

The introduction of the rechargeable battery has allowed manufactures to reduce the size of the IPG while increasing its service left. A non-rechargeable battery will last 2 to 5 years. A rechargeable battery will have a much longer left, at least 9 years. The Medtronic rechargeable system is unique in that it is designed to shut off and require replacement at 9 years $^{13)}$.

Medtronic's restore sensor IPG (Adaptive Stim ${ }^{\circledR}$ ) is the first neurostimulator to adjust stimulation automatically as the patient changes position. It also records data recording patient activity that clinicians can use to assess, evaluate, and optimize a patient's neurostimulation experience ${ }^{13)}$.

The Boston Scientific system employs multiple independent current control which could result in a more discrete distribution of the current in the neural structures than is available with the other IPGs. The Boston Scientific Spectra pulse generator is the only one offering 32 independent stimulation channals ${ }^{19)}$.

Nervo $^{\circledR}$ (Menlo Park, CA, USA) produces an IPG that can deliver stimulation up to $10,000 \mathrm{~Hz}^{19}$.

\section{5) Hard ware improvement and miniaturization}

As Slavin et al. ${ }^{23)}$ overviewed that the spinal cord stimulation in near future will most likely include introduction of rechargeable pulse generators, tool for minimally invasive implantation of paddle type electrode development of multicolumn stimulation, with 3, 4 and 5 column paddle leads, and the ability to connect multiple electrodes (with up to 32 active contacts) to a single generator, to name just a few.

\section{๑) Cervical spinal cord stimulation in prevention and treatment of cerebral vasospasm}

Cervical spinal cord stimulation can induce vasodilatation and increase cerebral blood flow. However, the pathophysiology of vasospasm after subarachnoid hemorrhage (SAH) may provide insight into the role of SCS in such conditions ${ }^{46}$.

Slavin et al. ${ }^{46)}$ hypothesized that effect of SCS on vasodilatation may be related to modulation of activity of phosphodiesterases 5 (PDE5) and nitric oxide synthase 1 (NOS1), resulting in enhancement of nitric oxide (NO) - cyclic guanosine monophosphate (cGMP) pathway, which may help prevent and/or treat vasospasm after SAH.

Investigations in animal experiments have clearly demonstrated augmentation of $\mathrm{CBF}$ with cervical $\mathrm{SCS}^{18,20)}$. SCS has been used to treat cerebral vasospasm after SAH in different animal models ${ }^{2,18)}$ and human patients ${ }^{37)}$. In this study, SCS electrodes were implanted and safely maintained for two-week period in the acute settings of aneurysmal SAH to prevent cerebral vasospasm from ruptured aneurysms. However, the scientific explanation of the CBF augmentation with SCS remains unclear. Other studies have shown that SCS can activate small-diameter sensory neurons expressing the transient receptor potential $\mathrm{V} 1$ through stimulating the large-diameter $A \beta$ fibers and spinal interneurons, resulting in increae in release of calcitonin gene-related peptide ${ }^{7,44)}$ and nitric oxide ${ }^{16)}$. Extra- 
cellular signal-regulated kinase (ERK), which is expressed in neurons located in the laminae I and II of the dorsal horn of spinal cord, is involved in intracellular signaling cascades ${ }^{48)}$, and is also important in the vasodilatation induced by $\operatorname{SCS}^{16}$. The experimental and initial clinical data do support the idea that upper cervical SCS facilitates CBF in patients with vasospasm and improves outcome ${ }^{37)}$. Moreover, it has been suggested that there are endogenous protective mechanisms, such as NOcGMP vasodilatation pathway, against vasospasm in the brain ${ }^{42)}$ and they may be activated by a preconditioning stimulus.

Based on these study results, we speculate that cervical SCS may function as a preconditioning stimulus for prevention and treatment of cerebral vasospasm induced by SAH. Also, it is hypothesized that SCS decreases PDE5 activity and increases expression level of eNOS (endothelial nitric oxide synthase), therefore enhancing NO-cGMP pathway and preventing vasospasm caused by SAH. The exact mechanism of action of SCS on vasospasm remains to be determined, and studies on PDE5 activity and eNOS expression level in the spinal cord and brain before and after cervical SCS in animal mode with SAH are necessary to support the hypothesis.

\section{7) High frequency alternating stimulation}

HF-SCS may offer several distinct benefits over traditional LF-SCS. The first is that the it may effectively target axial back pain, which often does not respond as well to traditional spinal cord stimulation. The second is that HF-SCS appears capable of delivering pain relief without inducing paresthesia. This latter observation has obvious benefits for patient from not having unpleasant induced paresthesia from stimulation, but also simplifies implantation of the system by eliminating the intraoperative paresthesia mapping, historically required to ensure proper lead positioning.

The observation that HF-SCS achieves pain relief without inducing paresthesia could represent an important finding which furthers our understanding of spinal cord stimulation's mechanisms of action ${ }^{40)}$.

Current hypothesis, based in part on "gate control theory" suggests that sensory stimulation is a necessary element to achieve pain control. However, studies involving HF-SCS suggest that spinal cord stimulation may be able to achieve pain relief without direct sensory fiber recruitment and the inducement of paresthesia. This would imply that additional neurophysiological mechanisms may be involved that are more responsive to stimulation at higher frequencies.

One hypothesized target is selective modification of wide dynamic range (WDR) neurons in the dorsal horn. Numerous studies have shown that after nerve damage or repeated stimulation, WDR neurons can develop hypersensitization ${ }^{3}$. The resulting increased firing pattern likely plays a key role in the pathophysiology of neuropathic pain.
Preclinical studies have shown that HF-SCS can modulate the firing pattern of WDR neurons in response to stimulus ${ }^{18)}$. Experiments were conducted in both rat and goat models. Direct microneurographic recording of WDR firing patterns in response to peripheral sensory stimulation were gathered. In these studies, noxious stimulation applied to the limbs of the test animal resulted in an expected increased firing pattern in the WDR neurons. When the test was repeated in the presence of HF-SCS, however, the WDR response was greatly attenuated.

Combining these preclinical findings with the clinical observation of paresthesia-free pain relief suggests that what may be unique about HF-SCS would be that the energy delivered is sufficient to modify the electrophysiological response of certain neurons (eg, WDR neurons) without triggering the outright activation of neurons that would lead to a sensory affect (eg, paresthesia) ${ }^{40)}$.

Stated another way, the distinction may be that HF-SCS modifies a neuron's firing response, whereas LF-SCS actually triggers a neuron to fire. If true, such findings could expand our understanding about the possible mechanisms of action involved with SCS, thereby allowing us to consider the intuitively logical notion that a better therapeutic effect can be achieved at stimulation parameters that do not necessarily trigger a sensory response ${ }^{40)}$. Clearly, additional work is warranted in this area to further explore these concepts.

Another procedural benefit associated with HF-SCS is that it can utilize standard, percutaneous leads. Although paddle leads paired with conventional SCS systems are showing improved results in some patients, the technique is more invasive, more complex, and less reversible than using percutaneous leads. As both platforms will undoubtedly play an important role in SCS therapy, a percutaneous-based system that offers superior results would likely prove simpler and faster for most pain centers ${ }^{40)}$.

The initial clinical observation that HF-SCS is able to achieve pain relief without paresthesia also holds important implications for our understanding of the mechanism of action for all spinal cord stimulation. The concept that stimulating at higher frequencies may allow a neuromodulatory effect (ie, altering when and how neurons respond to stimuli), without triggering a sensory effect (ie, actually forcing neurons to fire), could deepen our understanding of the neurophysiological mechanisms involved with SCS. Research in this area is clearly still in its nascency, but if confirmed, could open new areas of experimental design and exploration ${ }^{40)}$.

\section{8) Smart Patch}

The pain relieving action of electricity is usually explained in particular by two main mechanisms: first, segmental inhibition of pain signals to the brain in the dorsal horn of the spinal cord and second, activation of the descending inhibitory pathway 
with enhanced release of endogenous opioids and other neurochemical compounds (serotonin, noradrenaline, gamma aminobutyric acid (GABA), acetylcholine and adenosine). The modern electrotherapy of neuromusculo-skeletal pain is based in particular on the following types: transcutaneous electrical nerve stimulation (TENS), percutaneous electrical nerve stimulation (PENS or electro-acupuncture) and spinal cord stimulation (SCS). In mild to moderate pain, TENS and PENS are generally effective methods, whereas SCS is very useful for therapy of refractory neuropathic or ischemic pain. In 2005, high tone external muscle stimulation (HTEMS) was introduced. In diabetic peripheral neuropathy, its analgesic action was more pronounced than TENS application. HTEMS appeared also to have value in the therapy of symptomatic peripheral neuropathy in end-stage renal disease (ESRD). Besides its pain-relieving effect, electrical stimulation is of major importance for prevention of treatment of muscle dysfunction and sarcopenia. In controlled clinical studies electrical myostimulation (EMS) has been shown to be effective against the sarcopenia of patients with chronic congestive heart disease, diabetes, chronic obstructive pulmonary disease and ESRD.

Two different theories that can elucidate possible mechanisms of SCS-induced vasodilation for the application for ischemic vascular disorders. The first one implies that the SCS effect depends on inhibition of the sympathetic outflow transmitted via nicotinic receptors in the ganglia and acting mainly on a1-receptor at the nerve end origin junction ${ }^{6,45}$.

SCS applied at the spinal L1-L2 segments antidromically activates large diameter A $\beta$ DC (dorsal column) axons with collaterals to the superficial $\mathrm{DH}^{17,28)}$. Application of SCS at $\mathrm{T} 1-\mathrm{T} 2$, or at higher cervical levels, provides pain relief by reducing the frequency and to some extent also the severity of angina attacks, the intake of short-acting nitrates is also reduced $^{4,21,47)}$.

Studies have proposed a stimulation-induced flow increase or redistribution of blood supply where as others interpret the reduction of coronary ischemia (decreased ST changes, reversal of lactate production) at being mainly due to a decreased cardiomyocyte oxygen demand ${ }^{9,17)}$.

Recent studies indicate that SCS-induced local release of catecholamines in the myocardium may trigger protective changes related to mechanisms behind such ischemic preconditioning but without producing or masking signs of ischemic changes in the heart ${ }^{38)}$. There are also other signs indicating that SCS may induce a state similar to the following a short ischemic period- for example, by activating protein-kinase $\mathrm{C}$, a substance that is pivotal in ischemic preconditioning ${ }^{38)}$.

The "general common pathway" in the communication between the central nervous system and the heart is the intrinsic cardiac nerves (ICN). The ICN is located in the cardiac ganglionated plexuses covered by epicardial fat pads situated on the myocardium ${ }^{1,14)}$. The ICN responds to ischemic stress by a marked activity increase even if the ischemic region is situated far away from the neuronal population ${ }^{1,14)}$.

\section{CONCLUSION}

Advancement of new technological and conceptional development of spinal cord stimulation (i.e., new frequencies and stimulation paradigms, new devices, and new targets) have provided the physicians specializing pain the more in depth of knowledge and understanding about its usefulness and role in the management of chronic pain to relieve pain more efficiently, improve functional status and quality of life. Its development is still ongoing and is opening a new horizon for the treatment of patients with chronic, disabling pain of various causes.

\section{REFERENCES}

1. Armour JA, Linderoth B, Arora RC, DeJongste MJ, Ardell JL, Kingma JG Jr, et al.: Long-term modulation of the intrinsic cardiac nervous system by spinal cord neurons in normal and ischemic hears. Auton Neurosci 95(1-2):71-79, 2002

2. Bbel H, Schomacker K, Balogh A, Volz M, Funker J, Schicha $\mathrm{H}$, et al.: High cervical spinal cord stimulation (CSCS) increases regional cerebral blood flow after induced subarachnoid haemorrhage in rats. Minim Invasive Neurosurg 44:167-171, 2001

3. Baron R: Mechanisms of Disease: neuropathic pain-a clinical perspective. Nat Clin Practice Neurol 2(2):105-106, 2005

4. Buchser E, Durrer A, Albrecht E: Spinal cord stimulation for the management of refractory angina pectoris. J Pain Symptom Manage 31(4, Suppl):S36-S42, 2006

5. Craig $\mathrm{AD}$ : How do you feel? Interoception: the sense of the physiological condition of the body. Nat Rev Neurosci 3:655-666, 2002

6. Croom JE, Foreman RD, Chandler MJ, Barron KW: Cutaneous vasodilation during dorsal column stimulation is mediated by dorsal roots and CGRP. Am J Physiol 272(2 Pt 2):H950-H957, 1997

7. Croom JE, Foreman RD, Chandler MJ, Barron KW: Cutaneous vasodiation during dorsal column stimulation is mediated by dorsal roots and CGRP. Am J Physiol 272:H950-957, 1997

8. Cuellar JM, Alataris K, Walker A, Yeomans DC, Antognini JF: Effect of high-frequency alternating current on spinal afferent nociceptive transmission. Neuromodulation 16(4):318-327, discussion 327, 2013

9. Dejongst MJL, Haaksma J, Hautvast RW, Hillege HL, Meyler PW, Staal MJ, et al.: Effects of spinal cord stimulation on daily life myocardial ischemia in patients with severe coronary artery disease, A prospective ambulatory ECG study. Br Heart J 71:413418, 1994

10. De Ridder D, Plazier M, Kamerling N, Menovsky T, Vanneste S: Burst spinal cord stimulation for limb and back pain. World Neurosurg 80(5):642-649, e1, 2013

11. De Ridder D, Vanneste S, Plazier M, Van Loo E, Menovsky T: Burst spinal cord stimulation: toward paresthesia-free pain suppression. Neurosurgery 66:986-990, 2010

12. Levy RM: Spinal cord stimulation in 2020. Neuromodulation 16:93-96, 2013 
13. Falowski S, Celii A, Sharans: Spinal cord stimulation. An update. Neurotherapeutics 5:86-99, 2008

14. Foreman RD, Linderoth B, Ardell JL, Barron KW, Chandler MJ, Hull SS Jr, et al.: Modulation of intrinsic cardiac neurons by spinal cord stimulation: implications for its therapeutic use in angina pectoris. Cardiovasc Res 47(2):367-375, 2000

15. Frot M, Mauguiere F, Magnin M, Garcia_Larrea L: Parallel processing of nociceptive A-delta inputs in SII and midcingulate cortex in humans. J Neurosci 28:944-952, 2008

16. Goksel HM, Raradag O, Turaclar U, Tas F, Oztoprak I: Nitric oxide synthase inhibition attenuates vasoactive response to spinal cord stimulation in an experimental cerebral vasospasm model. Acta Neurochir (Wien) 143:383-390, 2001

17. Guan Y, Wacnik PW, Yang F, Carteret AF, Chung CY, Meyer RA, et al.: Spinal cord stimulation-induced analgesia: electrical stimulation of dorsal column and dorsal roots attenuates dorsal horn neuronal excitability in neuropathic rats. Anesthesiology 113(6):1392-1405, 2010

18. Gurelik M, Kayabas M, Karadag O, Goksel HM, Akyuz A, Topaktas S: Cervical spinal cord stimulation improves neurological dysfunction induced by cerebral vasospasm. Neuroscience 134 : 827-832, 2005

19. Barolat G, North RG: Spinal cord stimulation; equipment and implantation techniques. In; Kim J Burchiel, ed. Surgical management of Pain. New York Thieme 340-346, 2014

20. Isono M, Kaga A, Fujiki M, Mori T, Hori S: Effect of spinal cord stimulation on cerebral blood flow in cats. Stereotac Funct Neurosurgery 64:40-46, 1995

21. Jessurun GAJ, Dejongste MJL, Blanksma PK: Current views on neurostimulation in the treatment of cardiac ischemic syndromes. Pain 66(2-3):109-116, 1996

22. Kulkarni B, Bentley DE, Elliott R, Youell P, Watson A, Derbyshire SW, et al.: Attention to pain localization and unpleasantness discriminates the functions of the medial and lateral pain systems. Eur J Neurosci 21:3133-3142, 2005

23. Lam CK, Rosenow JM: Patient perspectives on the efficacy and ergonomics of rechargeable spinal cord stimulators. Neuromodulation 13:218-223, 2010

24. Lee JY, Huang DL, Keep R, Sagher O: Effect of electrical stimulation of the cervical spinal cord on blood flow following subarachnoid hemorrhage. J Neurosurg 109:1148-54, 2008

25. Lipov EG, Joshi JR, Slavin KV: Hybrid neuromodulation technique: use of combined spinal cord stimulation and peripheral nerve stimulation in treatment of chronic pain in back and legs. Acta Neurochir (Wien) 150:971, 2008

26. Lppez-Garcia JA, King AE: Membrance properties of physiologically classified rat dorsal horn neurons in vitro: correlation with cutaneous sensory afferent input. Eur J Neurosci 6:998-1007, 1994

27. Mannheimer C, Eliasson T, Andersson B,Bergh CH, Auqustinsson LE, Emanuelsson $\mathrm{H}$, et al.: Effects of spinal cord stimulation in angina pectoris induced by pacing and possible mechanism of action. Br Med J 307:477-480, 1993

28. Meyerson BA, Ren G, Herregodts P, Linderoth B: Spinal cord stimulation in animal models of mononeuropathy: effects on the withdrawal response and the flexor reflex. Pain 61(2):229-243, 1995

29. Mironer YE, Hutcheson JK, Satterthwaite JR, LaTourette PCl: Prospective, two-part study of the interaction between spinal cord stimulation and peripheral nerve field stimulation in pati- ents with low back pain: development of a new spinal-peripheral neurostimulation method. Neuromodulation 14:151-155, 2011

30. Moens M, Sunaert S, Mariën P, Brouns R, De Smedt A, Drooqmans $S$, et al.: Spinal cord stimulation modulates cerebral function: an fMRI study. Neuroradiology 54(12):1399-1407, 2012

31. Navarro RM, Vercimak DC: Triangular stimulation method utilizing combination spinal cord stimulation with peripheral subcutaneous field stimulation for chronic pain patients: a retrospective study. Neuromodulation 15:124-131, 2012

32. Ochoa JL, Torebjork HE: Paraesthesiae from ectopic impulse generation in human sensory nerves. Brain 103:835-853, 1980

33. Price DD: Psychological and neural mechanisms of the affective dimension of pain. Science 288:1769-1772,2000

34. Rainville P, Duncan GH, Price DD, Carrier B, Bushnell MC: Pain affect encoded in human anterior cingulate but not somatosensory cortex. Science 277:968-971, 1977

35. Rees H, Roberts MHT: Antinociceptive effects of dorsal column stimulation in the rat: involvement of the anterior pretectal nucleus. J Physiol 417:375-388, 1989

36. Ridder DD, Plazier M, Kamerling N, Menovsky T, Vannaste S: Burst Spinal Cord stimulation for limb and back pain. Word Neurosurgery 642-649, 2013

37. Slavin KV, Vannemereddy PS, Goellner E, Alaraj AM, Aydin S, Eboli P, et al.: Use of cervical spinal cord stimulation in treatment and prevention of arterial vasospasm after aneurysmal subarachnoid hemorrhage technical details. Neuroradiol J 24:131-135, 2011

38. Southerland EM, Milhorn DM, Foreman RD, Linderoth B, DeJonqste MJ, Armour JA, et al.: Preemptive, but not reactive, spinal cord stimulation mitigates transient ischemia-induced myocardial infarction via cardiac adrenergic neurons. Am J Physiol Heart Circ Physiol 292(1):H311-H317, 2007

39. Takanashi Y, Shinonaga M: Spinal cord stimulation for cerebral vasospasm as prophylaxis. Neurol Med Chir (Tokyo) 40:352357,2000

40. Vallejo R: High frequency spinal cord stimulation: An emerging treatment option for patients with chronic apin. Techniques in regional anesthesia and pain management 16:106-112, 2012

42. Van Buyten JP, AI-Kaisy A, Smet I, Palmisani S, Smith T: High frequency spinal cord stimulation for the treatment of chronic back pain patients: results of a prospective multicenter European clinical study. Neuromodulation 16(1):59-65, discussion 65-66, 2013

42. Vellimana AK, Milnor E, Azad TE, Harries ML, Gidday JM, $\mathrm{Han} \mathrm{BH}$, et al.: Endothelia nitric oxide synthase mediates endogenous protection against subarachnoid hemorrhage-induced cerebral vasospasm. Stroke 42:776-782, 2011

43. Whitehorn D, Burgess PR: Changes in polarization of central branches of myelinated mechanoreceptor and nociceptor fibers during noxious and innocuous stimulation of the skin. J Neurophysiol 36(2):226-237, 1973

44. Wu M, Komori N, Qin C, Farber JP, Linderoth B, Foreman $\mathrm{RD}$ : Roles of peripheral terminals of transient receptor potential vanilloid-1 containing sensory fibers in spinal cord stimulationinduced peripheral vasodilation. Brain Res 1156:80-92, 2007

45. Wu M, Linderoth B, Foreman RD: Putative mechanisms behind effects of spinal cord stimulation on vascular diseases: a review of experimental studies. Auton Neurosci 138(1-2):9-23, 2008

46. Yin D, Slavin KU: A hypothesis on possible neurochemical mechanisms of action of cervical spinal cord stimulation in prevention 
and treatment of cerebral arterial vasospasm after aneurysmal subarachnoid hemorrhage. Medical hypotheses 85:355-358, 2015 47. Yu W, Maru F, Edner M, Hellstrëm K, Kahan T, Persson H: Spinal cord stimulation for refractory angina pectoris: a retrospective analysis of efficacy and cost-benefit. Coron Artery Dis
15(1):31-37, 2004

48. Zhung ZY, Gerner P, Woolf CJ, JI RR: ERK is sequentially activated in neurons, microglia, and astrocytes by spinal nerve ligation and contributes to mechanical allodynia in this neuropathic pain model. Pain 114:149-159, 2005 